\title{
LC Headings from March-June 2019 Lists
}

Compiled by Ann Heinrichs

The new headings listed here reflect the most recent information available at the time of publication. Items in this list were selected from 2019 list numbers 03 (March 15), 04 (April 12), 05 (May 17), and 06 (June 14).

\section{SUBJECT HEADINGS}

150 Academic libraries--Services to foreign students [May Subd Geog] [sp2019000235]

550 BT Students, Foreign--Services for

150 Antisemitic literature [May Subd Geog] [sp2019002541]

450 UF Anti-Jewish literature

450 UF Anti-Semitic literature

550 BT Literature

680 Here are entered collections of works reflecting antisemitic views or policies as well as discussions of such works. This heading is not used for individual works, which are entered under headings appropriate to the content, genre, and/or form of the work.

680 Works that diminish the scale and significance of the Holocaust or assert that it did not occur are entered under Holocaust denial literature. Works about antisemitism as a theme in literature are entered under Antisemitism in literature.

681 Note under: Holocaust denial literature; Antisemitism in literature

150 Antisemitism in literature [Not Subd Geog] [sp 93008401]

680 Here are entered works on the theme of antisemitism in literature. Collections of works reflecting antisemitic views or policies as well as discussions of such works are entered under Antisemitic literature. ADD FIELD

681 Note under Antisemitic literature ADD FIELD

150 Archives--Administration [sp2007101328]

450 UF Archival administration ADD FIELD

450 UF Archives--Management ADD FIELD

150 Boyan Hasidim [May Subd Geog] [sp2019000510]

450 UF Boyana Hasidim

550 BT Hasidim

150 Christian fiction, Philippine (English) [May Subd Geog] [sp2015001744]

450 UF Christian fiction, English--Philippines

450 UF Philippine Christian fiction (English)

550 BT Philippine fiction (English) 
150 Christian literature, Samoan [May Subd Geog] [sp2018003259]

450 UF Samoan Christian literature

550 BT Samoan literature

150 Devotional poetry, French [May Subd Geog] [sp2019000827]

450 UF French devotional poetry

550 BT French poetry

150 Federal Day of Thanksgiving, Repentance, and Prayer (Switzerland) [May Subd Geog] [sp2018000441]

$450 \quad$ UF Bettag (Switzerland)

$450 \quad$ UF Digiuno federale (Switzerland)

450 UF Eidgenössischer Dank- Buss- und Bettag (Switzerland)

450 UF Jeûne fédéral (Switzerland)

$450 \quad$ UF Rogaziun federala (Switzerland)

550 BT Holidays--Switzerland

150 Five hindrances (Buddhism) [sp2019000168]

450 UF Nivaranas (Buddhism)

550 BT Buddhism--Doctrines

150 Gājana (Hindu festival) [May Subd Geog] [sp2018002971]

053 BL1239.82.G34

450 UF Gaajan (Hindu festival)

450 UF Gajan (Hindu festival)

550 BT Fasts and feasts--Hinduism

150 Holocaust denial literature [May Subd Geog] [sp 96009503 ]

680 Here are entered works that diminish the scale and significance of the Holocaust or assert that it did not occur. Works that discuss such assertions are entered under Holocaust denial. DELETE FIELD

680 Here are entered works that diminish the scale and significance of the Holocaust or assert that it did not occur. Works that discuss such assertions are entered under Holocaust denial. Collections of works reflecting antisemitic views or policies as well as discussions of such works are entered under Antisemitic literature. ADD FIELD

681 Note under Holocaust denial DELETE FIELD

681 Note under Holocaust denial; Antisemitic literature ADD FIELD

150 Icon painting--Macedonia (Republic) CHANGE HEADING

150 Icon painting--North Macedonia [sp 92004860 ]

150 Icon painting--Macedonia (Republic)--Ohrid CHANGE HEADING

150 Icon painting--North Macedonia--Ohrid [sp 89001906]

450 UF Icon painting--Yugoslavia--Ohrid (Macedonia) [Former heading] 
150 Icon painting--Macedonia (Republic)--Ohrid--Byzantine influences CHANGE HEADING

150 Icon painting--North Macedonia--Ohrid--Byzantine influences [sp 89001907]

551 BT Byzantine Empire--Civilization

150 Italian American Pentecostals [May Subd Geog] [sp2019000538]

450 UF Pentecostals, Italian American

550 BT Pentecostals--United States

150 Jewish bisexuals [May Subd Geog] [sp2019000135]

$450 \quad$ UF Bisexual Jews

$550 \quad$ BT Bisexuals

150 Jewish gays [May Subd Geog] [sp 89003905]

450 UF Gay Jews ADD FIELD

150 Macana, Our Lady of the [Not Subd Geog] [sp2019000650]

450 UF Macana, Virgen de la

450 UF Macana, Virgin of the

450 UF Nuestra Señora de la Macana

450 UF Our Lady of the Macana

450 UF Virgin of the Macana

450 UF Virgin of the Macana

500 BT Mary, Blessed Virgin, Saint--Apparitions and miracles--Mexico

500 BT Mary, Blessed Virgin, Saint--Devotion to--Mexico

151 Macedonia (Republic)--Civilization--Islamic influences CHANGE HEADING

151 North Macedonia--Civilization--Islamic influences [sp2012002886]

550 BT Islamic civilization

150 Middle class Muslims [May Subd Geog] [sp2019000414]

550 BT Muslims

150 Muslim school principals [May Subd Geog] [sp2019000432]

450 UF Islamic school principals

$550 \quad$ BT School principals

680 Here are entered works on school principals who are Muslim.

150 Muslims--Persecutions [May Subd Geog] [sp2018002969]

550 BT Persecution

150 Prayer in motion pictures [Not Subd Geog] [sp2018003037]

$550 \quad$ BT Motion pictures

150 Protestants--Religious identity [May Subd Geog] [sp2019000205]

450 UF Protestant identity

550 BT Identification (Religion) 
150 Rosh ha-Shanah [sp 85115451]

$450 \quad$ UF Day of Judgement ADD FIELD

450 UF Day of Judgment ADD FIELD

450 UF Day of Remembrance ADD FIELD

450 UF Judgement, Day of ADD FIELD

450 UF Judgment, Day of ADD FIELD

450 UF Remembrance, Day of ADD FIELD

450 UF Rosh Hashana ADD FIELD

450 UF Rosh Hashonoh ADD FIELD

450 UF Yom ha-Din ADD FIELD

450 UF Yom ha-Zikaron (Rosh ha-Shanah) ADD FIELD

450 UF Yom Ha-Zikkaron ADD FIELD

450 UF Yom Hadin ADD FIELD

450 UF Yom Hazikaron ADD FIELD

450 UF Yom Teruah ADD FIELD

450 UF Yom T'ruah ADD FIELD

150 Sacred music--Vietnam [sp2019001852]

150 Sadgora Hasidim [May Subd Geog] [sp2018003275]

450 UF Sadigorah Hasidim

450 UF Sadigura Hasidim

450 UF Sadigurah Hasidim

550 BT Hasidism

150 Slovak American Catholics [May Subd Geog] [sp2018003301]

$053 \quad$ BX1407.S55

450 UF Catholics, Slovak American

$550 \quad$ BT Catholics--United States

150 Sociobiology--Religious aspects--Confucianism [sp2019000405]

\section{GENRE/FORM TERMS}

155 Annotated bibliographies [gp2019026015]

555 BT Bibliographies

155 Ascension Day sermons [gp2019026010]

555 BT Occasional sermons

155 Ash Wednesday sermons [gp2019026009]

555 BT Lenten sermons 
155 Haggadot (Liturgical books) [gp2019026013]

455 UF Aggadahs (Liturgical books)

455 UF Aggadas (Liturgical books)

455 UF Aggadot (Liturgical books)

455 UF Aggadoth (Liturgical books)

455 UF Hagadot (Liturgical books)

455 UF Haggadahs (Liturgical books)

455 UF Haggadas (Liturgical books)

455 UF Haggados (Liturgical books)

455 UF Haggadoth (Liturgical books)

555 BT Liturgical books

155 Liturgical drama [gp2019026006]

455 UF Ecclesiastical drama

555 BT Religious drama

680 Medieval musical dramas performed during Roman Catholic liturgies.

155 Passover sermons [gp2019026012]

$555 \quad$ BT Occasional sermons

155 Rosh ha-Shanah sermons [gp2019026085]

455 UF Day of Judgement sermons

455 UF Day of Judgment sermons

455 UF Day of Remembrance sermons

455 UF Jewish New Year sermons

455 UF Rosh Hashana sermons

455 UF Rosh Hashanah sermons

455 UF Yom ha-Din sermons

455 UF Yom Ha-Zikkaron sermons

455 UF Yom Hadin sermons

455 UF Yom Hazikaron sermons

455 UF Yom Teruah sermons

455 UF Yom T'ruah sermons

555 BT Occasional sermons

\section{MEDIUM OF PERFORMANCE TERM}

162 psaltery [pp2013015578]

462 UF psalterion ADD FIELD 